\title{
The identification of post-acute patients at risk for hospital readmission: Clinical implications of the LACE index
}

\author{
Elizabeth Ritt*1, Nathan P. Taylor ${ }^{2}$ \\ ${ }^{1}$ Department of Nursing and Health, Benedictine University, Lisle, IL, United States \\ ${ }^{2}$ Symphony Post Acute Network, Lincolnwood, IL, United States
}

Received: April 21, 2016

DOI: $10.5430 /$ cns.v4n3p32

\author{
Accepted: June 5, 2016 \\ Online Published: June 8, 2016 \\ URL: http://dx.doi.org/10.5430/cns.v4n3p32
}

\begin{abstract}
Readmission to the hospital is stressful and disruptive for patients and accounts for billions of dollars in healthcare spending. The reduction of unplanned all-cause readmission to an acute care hospital is a major priority of the Centers for Medicare and Medicaid Services (CMS), policymakers, nurses, and all health care providers. Research studies have focused on patients discharged from an acute-care hospital to the home setting. The LACE index scoring tool has been used to predict the risk of death or unplanned readmission within 30 days after discharge from the hospital to the home setting. This descriptive, nonexperimental research study incorporated the LACE index in the record of patients admitted to two post-acute care facilities from surrounding acute care hospitals. Data were collected between September 2015 and February 2016. There were 164 participants in the study and ages ranged from 42-98 years of age with a mean age of 74.2. Of the 164 participants, a total of 19 participants, or $11.5 \%$, were readmitted to the hospital within a 30-day period after discharge. The most commonly identified comorbidities of diabetes without complications, congestive heart failure (CHF), chronic pulmonary disease, dementia, and cerebrovascular disease point to the layers of clinical complexity and risk when caring for older adults. This study supports further implementation of the LACE index with other clinical assessment tools that identify patients at greatest risk for an unplanned all-cause readmission to the hospital within 30 days of discharge to the post-acute care facility.
\end{abstract}

Key Words: Unplanned 30-day hospital readmission, Post-acute care facility, LACE index scoring tool

\section{INTRODUCTION}

The reduction of unplanned readmission ${ }^{[1]}$ to the hospital is of major importance to the Centers for Medicare and Medicaid Services (CMS), ${ }^{[1]}$ policymakers, nurses, and health care providers. Strides are being made in the reduction of hospital 30-day readmissions translating in an estimated 150,000 fewer hospital readmissions between January 2012 and December $2013^{[2]}$ which represents an eight percent reduction in the Medicare fee-for-service all-cause 30-day readmission rate. ${ }^{[2]}$ However, it is critical that all members of the interprofessional team are diligent in the development and implementation of new strategies that continue to reduce hospital readmission rates and corresponding costs.

Hospital readmissions are costly and in some instances unavoidable due to the severity of an illness or the serious progression of a chronic health condition. Hospital readmissions States. 
may be attributed to the lack of accessible and affordable health care, inadequate post-discharge and transitional care, patient non-compliance, lack of medication reconciliation, and less than optimal communication between providers and patients. Furthermore, behaviors that puts a patient's health at risk such as a lack of exercise, alcohol and tobacco use, and inadequate nutrition contribute to the development of chronic health conditions and early death. ${ }^{[3]}$ The ability to accurately predict hospital readmission risk is of great interest in the identification of patients that would benefit most from interventions focused on transitions in care. ${ }^{[4]}$

Readmission to the hospital is a source of stress and disruption for patients and caregivers and accounts for billions of dollars in Medicare spending. In 2013, a health policy brief published by the Robert Wood Johnson Foundation ${ }^{[5]}$ cited that according to CMS, historically about one in five Medicare patients discharged from a hospital are readmitted within 30 days. During 2011, 3.3 million adult hospital patients were readmitted within 30 days of discharge at a cost of $\$ 41.3$ billion and Medicare patients' comprised 58\% of the incurred costs. ${ }^{[6]}$

In this study, a hospital readmission experience ${ }^{[1]}$ was defined as an unplanned all-cause readmission ${ }^{[1]}$ to an acute care facility from the post-acute care facility within 30 days of discharge from the originating facility. As noted by Brooks ${ }^{[7]}$ exceptions may include: patients who are discharged and transferred to another hospital; those who leave against medical advice; and planned readmissions as in the case of additional testing and diagnostic procedures.

Literature on hospital readmissions have focused primarily on patients discharged from acute hospitals to the home setting. An emphasis on communication between patients, their caregivers, and members of the health care team promotes quality of care, enhanced coordination of services, and improved discharge planning. These efforts have helped to decrease the incidence of hospital readmission rates in some instances by 20 to 40 percent. ${ }^{[8]}$ For example, the LACE index ${ }^{[9]}$ scoring tool (LACE index) developed in Canada, has been used to predict the risk of death or unplanned readmission within 30 days after discharge from the hospital to the home setting. It documents the length of stay ("L"), ${ }^{[9]}$ which includes the day of admission and discharge; acuity of the admission ("A"), ${ }^{[9]}$ comorbidity of the patient as measured by the Charlson comorbidity index score ("C $C$ "), ${ }^{[9]}$ and emergency department use as measured by the number of visits made in the six months prior to hospital admission ("E"). ${ }^{[9]}$

van Walraven et al. ${ }^{[9]}$ conducted a prospective cohort study in which patient and admission variables were collected for 4,812 medical and surgical patients who were discharged to the community from 11 hospitals in Ontario. ${ }^{[9]}$ They reported that the outcome predicted by the LACE index ${ }^{[9]}$ was a reliable measure and clinically relevant. ${ }^{[9]}$ The LACE in$\mathrm{dex}^{[9]}$ was found to be moderately discriminative and very accurate for predicting the risk of early death or unplanned readmission after discharge from the hospital to the community. ${ }^{[9]}$ This study suggested that additional research that examines the impact of the LACE index ${ }^{[9]}$ with different patient populations in alternate settings would be valuable.

Tan et al. conducted a retrospective study at Singapore General Hospital which included 127,550 eligible patients who were admitted to one of the medical departments at the hospital over a four-year period and discharged to their homes. Findings demonstrated that the LACE index ${ }^{[9]}$ may be useful in the identification of patients at risk for readmission to hospitals in Asian countries. ${ }^{[10]}$

A retrospective study of 253 patients was conducted to externally validate the accuracy of using the LACE index ${ }^{[9]}$ to predict 30 day readmissions in patients experiencing congestive heart failure ${ }^{[11]}(\mathrm{CHF})$ exacerbation. Wang et al. ${ }^{[11]}$ found that the LACE index ${ }^{[9]}$ may be more useful in predicting the incidence of visits to the emergency department during the post discharge period. The ability to provide patients with valuable community health resources during the post discharge period may reduce the number of visits to the emergency department by this population.

McAlister et al. ${ }^{[12]}$ conducted a study of 24,373 older patients with extensive comorbidities common to the LACE index ${ }^{[9]}$ who received health care services in the year prior to their admission to the hospital. They found that ongoing follow-up by the same physician coupled with frequent contact with the patient after discharge were important indicators of risk for death or readmission to the hospital. ${ }^{[12]}$

A research study conducted with a total of 507 patients with a mean age of 85 in the United Kingdom ${ }^{[13]}$ (UK) found that the LACE index ${ }^{[9]}$ was a good predictor of death rather than of hospital readmission, ${ }^{[13]}$ which reflects the findings of van Walraven et al. ${ }^{[9]}$ Cotter et al. ${ }^{[13]}$ found that the LACE index ${ }^{[9]}$ was not useful for predicting 30 day readmission in older inpatients. ${ }^{[13]}$ Thus, they concluded that greater emphasis be placed on individual patient health factors when developing future readmission prediction tools.

A national study conducted by Dharmarajan et al. ${ }^{[14]}$ found that readmission diagnoses and timing after admission for heart failure, acute myocardial infarction, or pneumonia do not differ by hospital 30 day risk standardized readmission rates. ${ }^{[14]}$ Both acute myocardial infarction and heart failure are comorbidities included on the LACE index. ${ }^{[9]}$ The re- 
sults of this study indicate that high performing hospitals experience fewer admissions across the spectrum of readmission diagnoses throughout the post-discharge period. ${ }^{[14]}$ This may suggest that broad clinical strategies and timely nursing interventions may be more helpful in reducing hospital readmission rather than examining medical diagnoses for specified time periods after admission to the hospital.

In summary, these studies recommend additional research using the LACE index ${ }^{[9]}$ with different patient populations in alternate settings. To date, there is limited published work that investigates the use of the LACE index ${ }^{[9]}$ inpatients admitted for rehabilitation and therapeutic services in a postacute facility. The phrase "readmitted patient" was used throughout the study to refer to the group of patients that experienced readmission to an acute care hospital within 30 days of admission ${ }^{[1]}$ to the post-acute care facility. Thus, this research study incorporated the LACE index ${ }^{[9]}$ in patients transferred from the hospital to the post-acute care setting.

\section{Aims}

The aims of this study were to: (1) evaluate the effectiveness of the LACE index ${ }^{[9]}$ in the identification of patients at greatest risk for an unplanned all-cause readmission ${ }^{[1]}$ to the hospital within 30 days of discharge ${ }^{[1]}$ to a post-acute care facility; and (2) to examine the LACE index ${ }^{[9]}$ scores among post-acute patients who experienced hospitalization within a 30-day period after discharge.

\section{MethodS}

\subsection{Design}

A descriptive, non-experimental design was selected since it was appropriate to the setting, efficient, and economical to manage. The design facilitated implementation of the LACE index ${ }^{[9]}$ so that it was non-disruptive to the workflow in the post-acute setting. The LACE index ${ }^{[9]}$ appeared in the same electronic system currently used by members of the clinical team with careful consideration given to time for completion of the tool. The electronic medical record ${ }^{[15]}$ provided comprehensive and enhanced real-time clinical information that was collected and analyzed in aggregate. ${ }^{[16]}$

\subsection{Instrument}

van Walraven et al. found that the LACE index ${ }^{[9]}$ was discriminative (C statistic 0.684 ) and very accurate (HosmerLemeshow goodness-of-fit-statistic $14.1, p=.59$ ) at predicting outcome risk. ${ }^{[9]}$ The methods used to derive the LACE index ${ }^{[9]}$ were both valid and transparent. The discrimination of the LACE index ${ }^{[9]}$ was better than that of the widely used Framingham score ${ }^{[17]}$ in many populations, which suggests that the LACE index ${ }^{[9]}$ is useful when applied to individual patients. ${ }^{[17-19]}$

\subsection{Participants}

A convenience sample of 164 participants records were selected based on criteria entered into the patient medical record intake referral management module. ${ }^{[20]}$ The LACE index ${ }^{[9]}$ was completed upon admission of every participant to the post-acute care facility transferred from an acute care hospital over a five month period. Participants ranged in age between 42-98 years with a mean age of 74.2. Eightyfive $(n=85)$ of the participants were females and 79 were male. A total of 87 participants reported race as African American/Black $(n=87)$, Caucasian/White $(n=60)$, Hispanic/Latino $(n=7)$, Asian $(n=4)$, and six participants did not disclose their race as presented in Table 1.

Table 1. Gender and race

\begin{tabular}{ll}
\hline Characteristic & Number (Percentage) \\
\hline \multirow{2}{*}{ Gender } & $79(48 \%)$ Male \\
& $85(52 \%)$ Female \\
& $87(53 \%)$ African/American \\
& $60(36.6 \%)$ Caucasian/White \\
Race & $7(0.043 \%)$ Hispanic/Latino \\
& $4(0.024 \%)$ Asian \\
& $6(0.037 \%)$ Did not disclose \\
\hline
\end{tabular}

\subsection{Data collection}

The study was conducted in two facilities that comprise a network of 32 post-acute facilities that operates throughout northern and central Illinois, northern Indiana, and central Arizona. Each patient admitted from an acute care hospital to the post-acute care facility to receive rehabilitation and therapy services was eligible to participate in the study regardless of the payer. The data collected focused on four areas: hospital length of stay, indication of emergency department admission, comorbidities (i.e. diagnosis code list), and the number of visits to the emergency department over the past six months. ${ }^{[9]}$ This information is typically available when a patient is discharged from a hospital that provides acute care services. However, it is usually not collected in a standard format, such as the LACE index, ${ }^{[9]}$ during the referral and transfer process.

The records of 164 patients admitted to one of the two facilities between of the months of September 2015 to January 2016 were analyzed in this study. The LACE index ${ }^{[9]}$ was programmed into the patient's medical record intake referral management module and served as the standardized method for receiving information from the referring acute care hospital. The LACE index ${ }^{[9]}$ risk score of hospital readmission was as follows: 0-4 low, 5-9 moderate, and 9 or greater was considered high risk. As part of the routine hospital transfer 
and referral process, the LACE index ${ }^{[9]}$ was completed at the post-acute care facility.

A training module was developed and posted on the intranet site which was made available within the patient record. In addition, the researchers conducted in-person training with each health care team member to foster the correct use of the LACE index. ${ }^{[9]}$ Periodic review of the data generated by the LACE index ${ }^{[9]}$ was monitored and validated for accuracy and completeness by the researchers. During periodic reviews, an initial validation was completed to verify that data was properly collected as well as to check that data collected matched the documentation in the patient's record. ${ }^{[15]}$ The researchers had access to individual participant data for the purpose of checking for accuracy and completeness of the LACE index. ${ }^{[9]}$ The final analysis and presentation of results are provided in the aggregate to adhere to confidentiality regarding participant information.

Adoption of the LACE index ${ }^{[9]}$ was continually monitored throughout the data collection period via automatic daily reports distributed to the researchers. Based on historical data, the researchers were able to estimate the number of LACE indexes ${ }^{[9]}$ that should be completed each week. Any delinquencies or discrepancies in collection quotas and errors were brought to the attention of the health care team and corrected immediately.

\subsection{Ethical considerations}

The researchers obtained institutional approvals from institutional review board and general counsel. Permission to use the LACE index ${ }^{[9]}$ was granted in writing. The researchers had access to participant data for the purpose of data collection and results were analyzed and presented in aggregate.

\subsection{Data analysis}

The data analysis reporting structure was built using Microsoft SQL Server Reporting Services (SSRS) in conjunction with a report manager application that provided daily automated reports. The data were analyzed using data reported from Microsoft SQL Server 2012 SP 2 version 11.0.5343.0012. At the end of each week of data collection, the LACE indexes were reviewed for completeness by the researchers. In addition, the research team reviewed the electronic medical record of each participant in the PointClickCare system which provided real-time views of patient information that allowed the researchers to verify whether or not the LACE index ${ }^{[9]}$ accurately reflected the medical diagnoses, treatment, and admission status for each participant. The information was aggregated and analyzed for consistency between the admission data and relevant clinical findings. After the initial analysis, the data were further analyzed using Tableau version 9.3. Tableau provides quick and efficient visualization of research data without the need to build sophisticated applications. ${ }^{[21]}$

\section{Results}

The risk for hospital readmission within a 30 day-period ${ }^{[1]}$ indicated that 119 participants $(72.5 \%)$ were at high risk, $44(26.8 \%)$ were considered to be at moderate risk, and one participant was deemed to be at low risk, as illustrated in Figure 1.

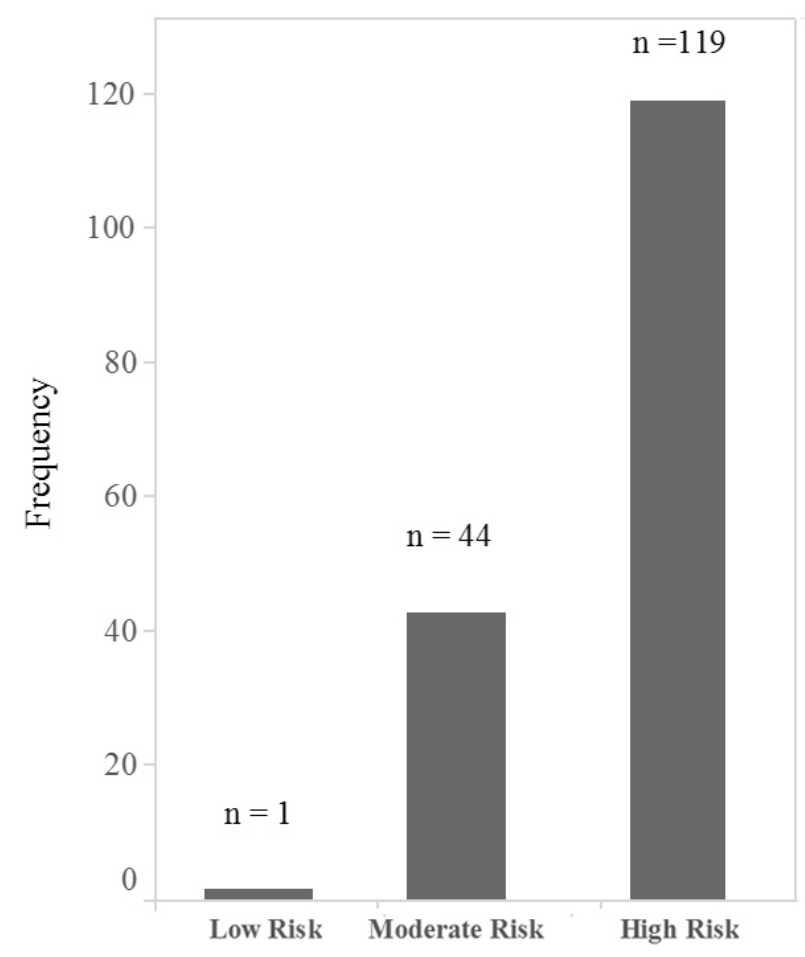

Figure 1. Frequency of LACE index ${ }^{[9]}$ category

The hospital length of stay ${ }^{[9]}$ before being admitted for postacute care ranged from 1-14 or more days as displayed in Figure 2.

Acuity ${ }^{[9]}$ reflects whether or not the patient was admitted to the hospital from the emergency department. In this study, $151(92 \%)$ of the participants were admitted to the emergency department and $13(8 \%)$ were admitted from home or another non-acute care facility. The Charlson comorbidity index ${ }^{[9]}$ score represents 14 medically diagnosed conditions. Figure 3 illustrates the frequency of all 14 comorbidities among the participants. The most frequently reported comorbidities included diabetes without complications $(\mathrm{n}=55), \mathrm{CHF}(\mathrm{n}=$ $38)$, chronic pulmonary disease $(n=33)$, dementia $(n=29)$, and cerebrovascular disease $(n=23)$.

Of the 164 participants in this study 19 (11.5\%) experienced an unplanned all-cause readmission ${ }^{[1]}$ to the hospital within a 
30 day period due to a broad range of medical complications and abnormal laboratory results. Of the 19 participants readmitted to the hospital, 16 were considered at high risk and three were considered at moderate risk for readmission according to the score on the LACE index. ${ }^{[9]}$ Hospital length of stay prior to admission to the post-acute care setting ranged from 3-14 or more days.

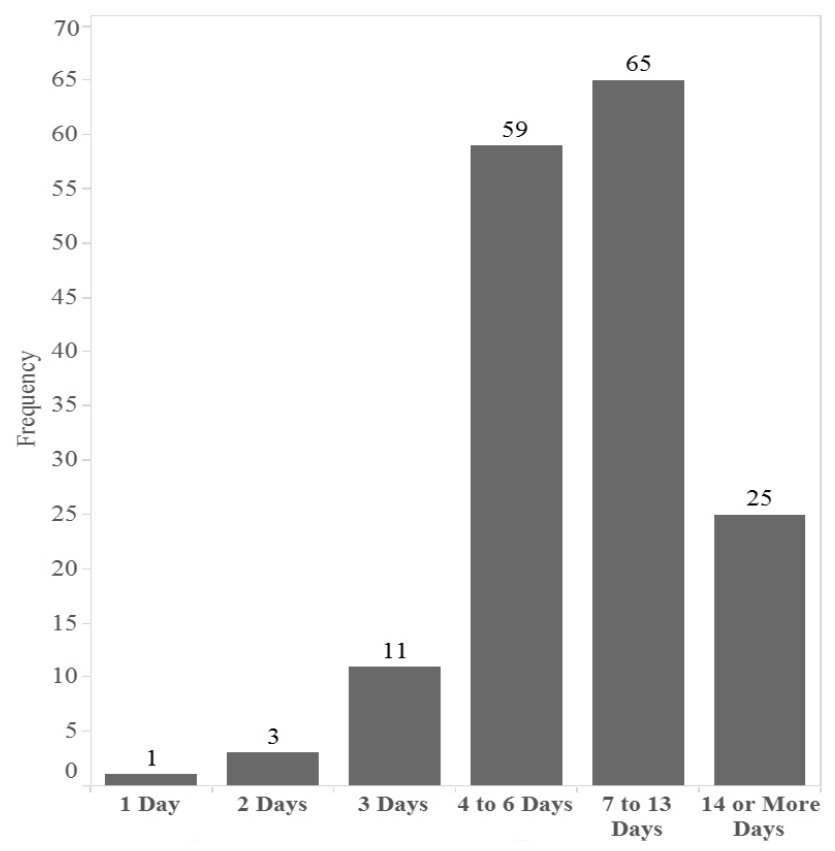

Figure 2. Hospital length of hospital stay ${ }^{[9]}$ (L)

Acuity of admission indicated that all 19 participants were admitted to the hospital via the emergency department. The most frequently reported Charlson comorbidities ${ }^{[9]}$ included diabetes $(n=12)$, CHF $(n=6)$, and previous myocardial infarction $(n=4)$. Of the 19 participants who were readmitted as patients to the hospital, 18 had a diagnosis of essential hypertension and 17 had a diagnosis of cognitive communication deficit. Visits to the emergency department within a six-month period prior to hospitalization excluding the current admission indicated that four of the 19 readmitted participants visited the emergency department at least once in the last six months.

\section{Discussion}

The majority of participants $(99.3 \%)$ in this study were deemed to be at moderate to high risk for an unplanned all-cause readmission ${ }^{[1]}$ to the hospital within 30 days. The 19 participants who did experience a readmission were all considered to be at moderate to high risk for readmission according to the LACE index. ${ }^{[9]}$ The LACE index ${ }^{[9]}$ could be implemented at the time of admission to alert nurses and other members of the interprofessional team that a newly admitted patient is at risk for readmission. Thus, these patients could be clinically monitored more closely for changes in their health status and early interventions may prevent or minimize an unplanned readmission to the hospital. This has direct implications for nurses and case managers who are monitoring patients throughout the post-acute stay.

The identification of the most frequently reported Charlson index ${ }^{[9]}$ comorbidities of chronic pulmonary disease, diabetes without complications, CHF, cerebrovascular disease, and dementia, point to the need for targeted and multifaceted nursing strategies and interventions that address the chronic and complex health care needs of patients. The additional identification of essential hypertension and cognitive communication deficit in the majority of readmitted patients may warrant additional attention to these conditions and the requisite coordination of care in the post-acute population. Tinker ${ }^{[22]}$ noted that comorbidities add multiple layers of complexity which are typically not evident when examining one chronic health condition. For example, patients may experience a potential drug interaction as a result of a new medication. ${ }^{[22]}$ This has direct implications for nurses and caregivers because they have the most contact with patients and are in the best position to assess comorbidities, plan and coordinate care, and evaluate patient outcomes.

It is a common practice in skilled and post-acute care facilities to transfer patients to hospitals when physicians and nursing staff determine the need for urgent services. ${ }^{[23]}$ Transfers between health care facilities increase the risk of harm and the potential of unfavorable patient outcomes that may result in costly hospitalizations. ${ }^{[24]}$ During this study, the readmission rate was significantly lower than the prior two years. Of the total number of participants, $11.5 \%$ experienced a hospital readmission which was well below the post-acute care network average of $19.4 \%$ (2014) and $18.1 \%$ (2015). ${ }^{[25]}$ This resulted in a cost savings to providers and payers as well as a decrease in the potential risk to patients during the hospital readmission process. The researchers suggest further use of the LACE index ${ }^{[9]}$ in the post-acute care setting over a longer period of time to evaluate whether or not the difference in the readmission rate noted in this study was related to the heightened awareness of the interprofessional team during the use of the LACE index ${ }^{[9]}$ or other clinical and admission factors.

The monitoring of hospital readmission continues to be an important clinical, financial, and policy area for health care providers. Recent legislative acts that protect access to Medicare $^{[26]}$ and focus on improving post-acute care ${ }^{[27]}$ require hospital readmission measures for skilled nursing facilities. The former requires value-based purchasing to use an all- 
cause hospital readmission measure as an initial performance measure, and the latter requires development of a potentially preventable readmission measures for skilled nursing facilities. ${ }^{[28]}$

Limitations of this study included a relatively small sample ( $n=164)$ in contrast to other studies conducted using the LACE index. ${ }^{[9]}$ Second, the LACE index ${ }^{[9]}$ has not been tested for reliability and validity for use in patient populations receiving rehabilitation and therapeutic services. Third, the ability to access patient information during the transfer process was not consistently available to the health care team. This necessitated follow-up phone calls and document review in order to obtain required patient information and data that needed to be entered into the LACE index ${ }^{[9]}$ scoring tool.

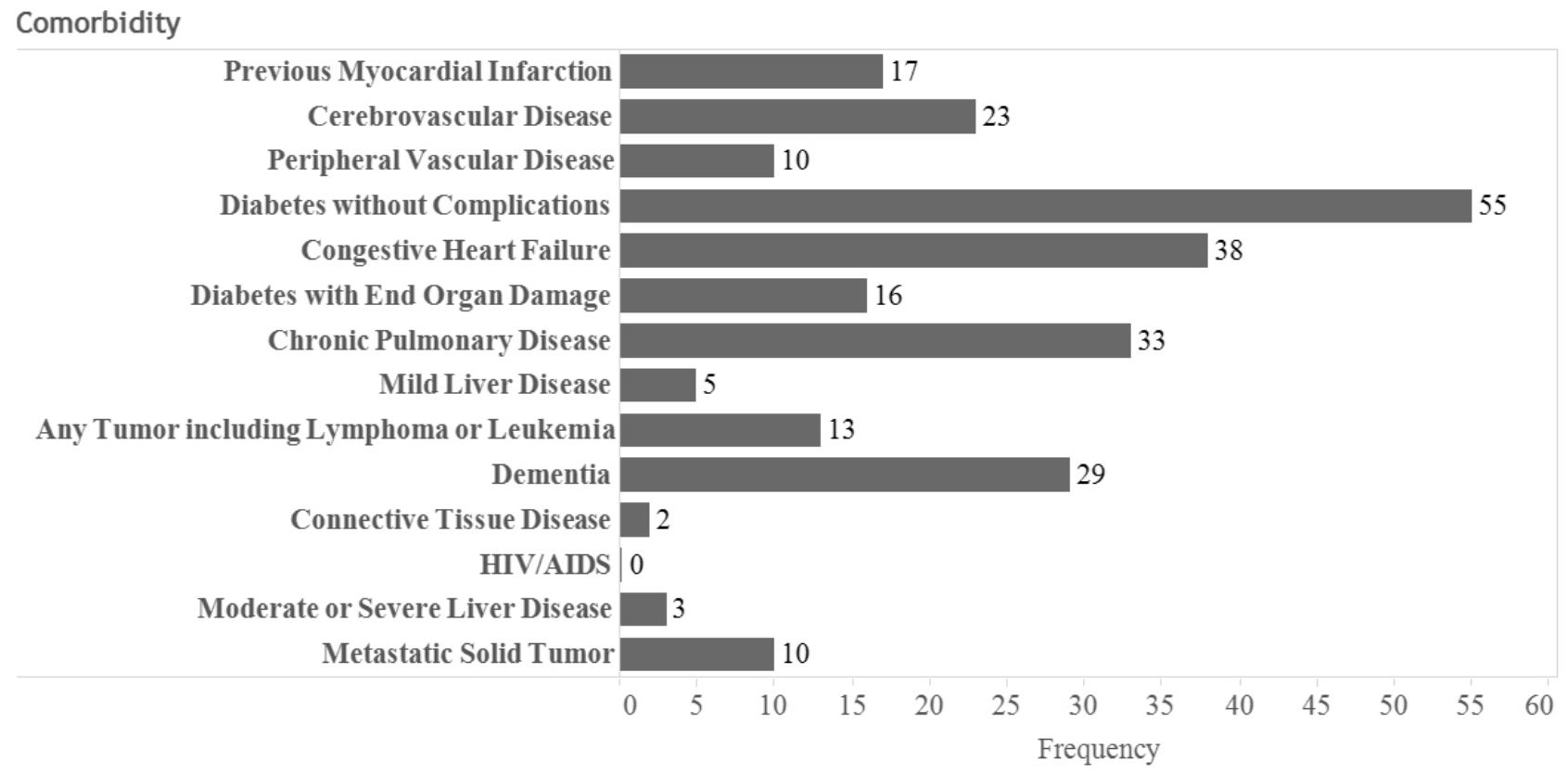

Figure 3. Frequency of comorbidities

Visits to the emergency department within the past six months indicated that 9 participants made three visits, 18 made two visits, and 137 did not access emergency services within the past six months

5. Conclusions and Relevance to ClinICAL PRACTICE

Future research regarding patients who are readmitted to the hospital from long-term care, skilled, and post-acute facilities is imperative. Readmission to the hospital is disruptive to the patient and caregivers, poses issues with coordination of care, and results in increased health care spending. The findings of this study support further exploration using the LACE index ${ }^{[9]}$ in conjunction with other appropriate clinical assessment tools and nursing care interventions in an effort to promote positive patient outcomes. The most common comorbidities of diabetes without complications, CHF, chronic pulmonary disease, dementia, and cerebrovascular disease noted in this study point to the layers of clinical complexity when caring for older adults. Furthermore, essential hypertension and cognitive communication deficit were fre- quent medical diagnoses among the readmitted patients and thus, may warrant additional attention and evaluation in the post-acute care setting.

Preventing or reducing hospital readmissions requires a comprehensive approach that includes clinical and predictive tools such as the LACE index ${ }^{[9]}$ in conjunction with careful examination of nursing staffing levels, acuity of patients, work environment, and educational and experiential preparation of providers of health care. Coordination of care during transition, effective communication among all stakeholders, valid and reliable admission assessment tools, and collaboration with patients and family members is critical in the prevention of hospital readmissions.

\section{Conflicts of InTERest Disclosure}

The authors declare they have no conflict of interest. 


\section{REFERENCES}

[1] Horwitz H, Partovian C, Lin Z, et al. Hospital-wide (all-condition) 30day risk-standardized readmission measure. Draft measure methodology report submitted by Yale New Haven Health Services Corporation/Center for Outcomes Research \& Evaluation. Contract number: HHSM-500-2008-0025I/HHSM-500-T0001, Modification No 000005. Prepared for Centers for Medicare \& Medicaid Services (CMS). 2011.

[2] U.S. Department of Health and Human Services. New HHS data shows major strides made in patient safety, leading to improved care and savings. Washington, DC; 2014, Available from: http://innovation.cms.gov/Files/reports/patie nt-safety-results.pdf

[3] Centers for Disease Control and Prevention. Chronic diseases and health promotion. Atlanta, GA. Available from: http://www.cdc gov/chronicdiseasse/overview/index.htm

[4] Kansagara D, Englander H, Salanitro A, et al. Risk prediction models for hospital readmission: systematic review. JAMA. 2011; 306 (15): 1688-1698. PMid: 22009101. http://dx.doi.org/10.1001/j ama. 2011.1515

[5] Robert Wood Johnson Foundation. Health Policy Brief: Medicare hospital readmissions reduction program. To improve care and lower costs, Medicare imposed a financial penalty on hospitals with excess readmissions. Princeton, NJ: Robert Wood Johnson Foundation; 2013. Available from: www healthaffairs.org

[6] Hines AL, Barrett M, Jiang J, et al. Conditions with the largest number of adult hospital readmissions by payer, 2011. Rockville, MD: Agency for Healthcare Research and Quality; 2014 Apr. Available from: http://medpac.gov/documents/reports/June07-Ent ireReport.pdf

[7] Brooks JA. Reducing hospital readmissions. American Journal of Nursing. 2015; 115(1): 62-65. PMid: 25545534. http://dx.doi .org/10.1097/01.NAJ .0000459639.76280.ae

[8] Naylor MD, Aiken LH, Kurtzman ET, et al. The importance of transitional care in achieving health reform. Health Affairs. 2011; 30(4): 746-754. PMid: 21471497. http://dx.doi.org/10.1377/hlt haff.2011.0041

[9] van Walraven C, Dhalla IR, Bell C, et al. Derivation and validation of an index to predict early death or unplanned readmission after discharge from hospital to the community. Canadian Medical Association Journal. 2010; 182(6): 551-557. http://dx.doi .org/10 . $1503 / \mathrm{cmaj} .091117$

[10] Tan SY, Low LL, Yang Y, et al. Applicability of a previously validated readmission predictive index in medical patients in Singapore: a retrospective study. BioMed Central Health Services Research. 2013; 13(366): 2949. http://dx.doi.org/10.1186/1472-696 3-13-366

[11] Wang H, Robinson RD, Johnson C, et al. Using the LACE index to predict hospital readmissions in congestive heart failure patients. BioMed Central Cardiovascular Disorders. 2014; 14(1): 1-8. http://dx.doi.org/10.1186/1471-2261-14-97

[12] McAlister FA, Youngson E, Bakal JA, et al. Impact of physician continuity on death or urgent readmission after discharge among patients with heart failure. CMAJ. 2013; 185(14): E681-689. PMid: 23959284. http://dx.doi.org/10.1503/cmaj.130048

[13] Cotter PE, Bahalla VK, Wallis SJ, Biram RW. Predicting readmissions: poor performance of the LACE index in an older UK pop- ulation. Age and Ageing. 2012; 41: 784-789. PMid: 22644078. http://dx.doi.org/10.1093/ageing/afs073

[14] Dharmarajan K, Hsieh AF, Lin Z, et al. Hospital readmission performance and patterns of readmission: retrospective cohort study of Medicare admissions. BMJ. 2013; 347: f6571. PMid: 24259033. http://dx.doi.org/10.1136/bmj.f6571

[15] Gunter TD, Terry NP. The emergence of national electronic health record architectures in the United States and Australia: models, costs, and questions. Journal of Medical Internet Research. 2005; 7(1): e3. PMid: 15829475. http://dx.doi.org/10.2196/jmir.7.1.e3

[16] Miriovsky BJ, Shulman LN, Abernethy AP. Importance of health information technology, electronic health records, and continuously aggregating clinical effectiveness research and learning health care Journal of Clinical Oncology. 2012; 30(34): 4243-4248. PMid: 23071233. http://dx.doi.org/10.1200/JC0.2012.42.8011

[17] Guzder RN, Gatling W, Mulle MS, et al. Prognostic value of the Framingham cardiovascular risk equation and the UKPDS risk engine for coronary heart disease in newly diagnosed type 2 diabetes: results from a United Kingdom study. Diabetes Medicine. 2005; 22(5): 554-62. PMid: 15842509. http://dx.doi.org/10.1111 /j.1464-5491.2005.01494.x

[18] Empan JP, Ducimetiere P, Arveiler D, et al. Are the Framingham and PROCAM coronary heart disease risk functions applicable to different European populations? The PRIME Study. European Heart Journal. 2003; 24(21): 1903-11. http://dx.doi.org/10.1016/j .ehj.2003.09.002

[19] Orford JL, Sesso HD, Stedman M, et al. A comparison of the Framingham and European Society of Cardiology coronary heart disease risk prediction models in the normative aging study. American Heart Journal. 2002; 144(1): 95-100. PMid: 12094194. http: //dx.doi.org/10.1067/mhj.2002.123317

[20] Hall H, Roussel L. Evidence-based practice. Jones and Bartlett Learning. 2014; 6-8. http://dx.doi .org/10.1037/14187-004

[21] Lee J, Oniki TA, Holmen JR, et al. A Comparative analysis of clinical data visualization development strategies: build vs. buy. Visual analytics in healthcare workshop. Washington, DC: AMIA Annual Symposium; 2014. 2.

[22] Tinker A. How to improve patient outcomes for chronic diseases and comorbidities. Health Catalyst. 2014; 1-5.

[23] Saliba D. Appropriateness of the decision to transfer nursing facility residents to the hospital. Journal of American Geriatrics Society. 2000; 48(2): 154-163. http://dx.doi.org/10.1111/j.1532-5 415.2000.tb03906. $\mathrm{x}$

[24] Assistant Secretary for Planning and Evaluation (ASPE). Hospitalization of nursing home residents: background and options. 2011.

[25] Symphony Post Acute Network. Yearly 30-day readmission rate report. Lincolnwood IL. April 14.

[26] Office CB. H.R. 4302. Protecting Access to Medicare Act of 2014 Available from: https://www.govtrack.us/congress/bills $/ 113 / \mathrm{hr} 4302$

[27] Library WE. Improving Medicare Post-Acute Care Transformation Act of 2014 (H.R. 4994; 113 ${ }^{\text {th }}$ Congress). Available from: https ://www . govtrack . us/congress/bills/113/hr4994

[28] U.S. Department of Health and Human Services, Office of Inspector General. Medicare nursing home resident hospitalization rates merit additional monitoring. Washington, DC; 2013. OEI-06-11-00040. 